\title{
An intelligent monitoring method and its experiment for bridge bearing
}

\author{
Qiang Liü ${ }^{1, a}$, Guangming $\mathrm{Li}^{1, \mathrm{~b}^{*}}$, Ruijuan Jiang ${ }^{2, \mathrm{c}}$, \\ Fang $\mathrm{Yu}^{2, \mathrm{~d}}$ and Weiming $\mathrm{Gai}^{2, \mathrm{f}}$ \\ ${ }^{1}$ School of Mechanical, Electrical and Information Engineering, Shandong University, \\ 180 Wenhua Xilu, Weihai, China 264209 \\ ${ }^{2}$ Shenzhen Municipal Design \& Research Institute Co., Ltd., 3007 Sungang Xilu, Shenzhen, \\ China 518029 \\ aliuqiangwh@126.com, bgmli@sdu.edu.cn, cjiangrj@hotmail.com, dyu_fang_03@163.com, \\ 'gaiwm@szmedi.com.cn
}

Keywords: structural health monitoring; virtual instrument; bridge bearing; data acquisition Abstract. The bridge bearing stress and deformation can eflect the safety of the bridge structure significantly. For bridge bearing status monitoring, identification and early warning technology, in recent years, is an important research direction for structural health monitoring. This paper is based on a model pot rubber bearing, combines with the practical application of bridge bearing condition, chooses specific sensors as data acquisition nodes, designs and develops the signal adjusting module, and uses the data acquisition card to build the hardware platforms of the data acquisition system. Furthermore here also uses virtual instrument technique to develop the the upper computer of this data acquisition system. The system is validated by performing laboratory loading test on a model bridge bearing. The experiment result and data are analyzed

\section{Introduction}

When the bridge major component suffered overload, impact or other effect, the strength and stiffness of the bridge structure deteriorate obviously, the spatial distribution of the bridge load will change [1,2], bridge bearing as the main connecting component between bridge superstructure and bridge understructure, its internal stress distribution will have evident changes. The internal stress of the bridge bearing can represent the condition of the bridge bearing and the safety of the whole bridge. Strengthening of bridge bearing health monitoring plays an important role in the assessment of the bridge integral safety [3,4].

There are some preliminary explorations in bridge bearings monitoring field. China Academy of Railway Sciences presented a three-direction force-measuring bearing [5]. It is on the basis of conventional bearing by integrated measuring force components, data acquisition and data processing equipment. But due to the reason that bearing can only measurement the counter force on three directions, it does not reflect the spatial distribution of bearing stress, and this bearing has strong applicability on the railway bridge only. Literature [6] introduced a adjustable hydraulic type pot rubber bearing and its internal with a oil cavity, using internal pressure will make the beams jacked up in a suspended state, calculating the bearing reaction force through measuring the oil pressure in real-time calculating, but the bearing only achieves vertical force measurements, mainly used to adjust the height of bearing. Sung-Inching and Nam-Sik Kim in Korea Pusan National University developed a technical proposal for measuring vertical displacement and load of the bearing, this technical proposal is based on Fiber Bragg grating sensor [7], but the bearing is not applied to engineering for a long time, its reliability has not been established. Dong-Ho Ha, department of civil engineering, Konkuk University, who raised putting piezoelectric materials in bridge bearing interior to monitor vehicle flowrate and bearing stress [8], with not much in-depth analysis of bearing internal stress distribution. Literature [9] introduced a way that PVDF sensor embedded in the laminated rubber bearing to monitor the stressing changes of the bearing, and assessment of bridge structure dynamic characteristics in real time. But PVDF material will affect the overall performance of the bearing. 
This paper proposes a method for monitoring the stress distribution in bridge bearing directly. Comparing with existing technical protocols, monitoring the mechanic property and eccentric load can more directly identify the health status of bearing and bridge more precisely

\section{The hardware component of bridge bearing monitoring system}

The framework of the hardware component of bridge bearing monitoring system is shown in Fig. 1. Data acquisition nodes is research on the structure and performance about sensors to determine how to layout the sensors in bearing, then through the signals adaptor module transform the initial voltage response to standard voltage volume (this process including voltage-stabilized source drive, signal conditioning and the anti-interference processing) and send the data acquisition card, the data acquisition card caches signals and sends them to the computer systerm through universal serial bus for processing.

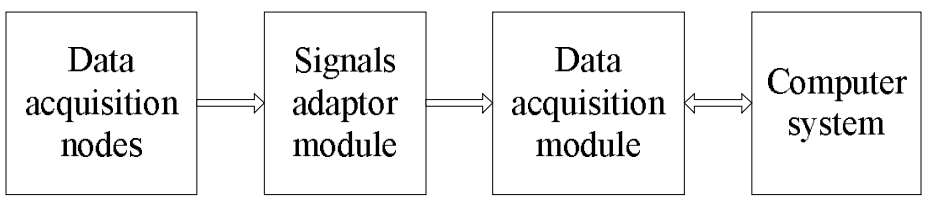

Fig. 1 The hardware component of bridge bearing monitoring system

This system uses Flexiforce A201 thin film piezoresistive sensor as shown in figure Fig. 2, and the essence of the sensor is a flexible printed circuit. The power amplifier circuit is shown in figure Fig. 3, which is suitable for this sensor.

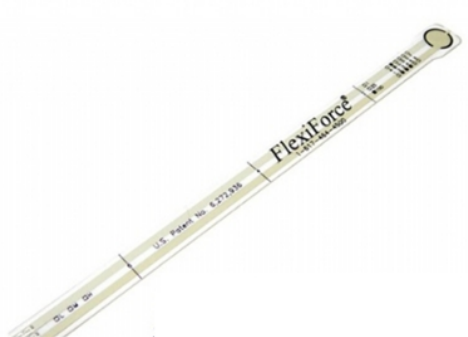

Fig. 2 Flexiforce A201

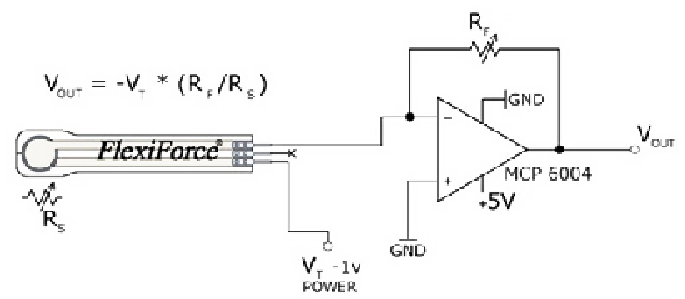

Fig. 3 The power amplifier circuit

Using Advantech USB4716 data acquisition card provides users with complete DDL driving and third-party driver support, as the data acquisition module. Voltage signal is single-ended input into AI0 AI15 ports from signal conditioning module, for analog signal-input data acquisition. This system uses MEAN WELL NES-100-5 DC switching power supply. The physical hardware connection is shown in Fig. 4.

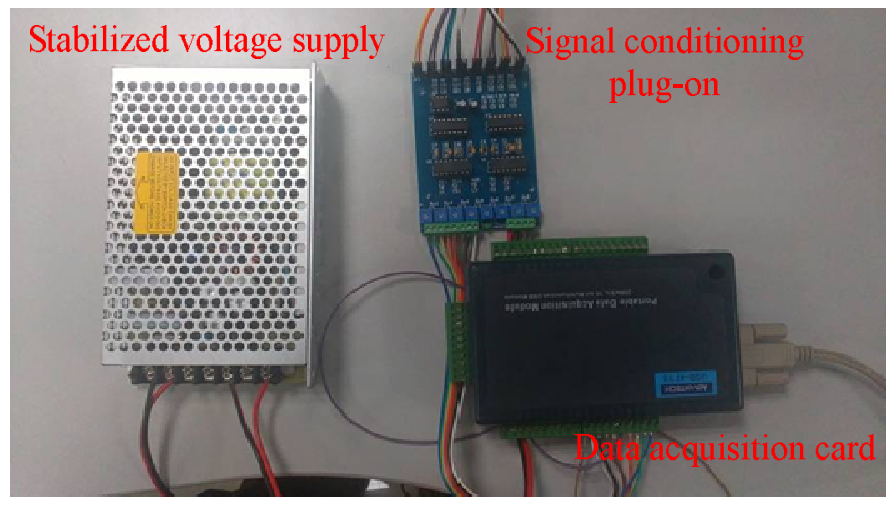

Fig. 4 The physical hardware connection 


\section{The upper computer of the bridge bearing monitoring system}

This paper, uses the virtual instrument technology combined with hardware modules. The system is developed by using visual graphics programming language, based on the LABVIEW development platform. On this development platform, program runs as the form of data flow. The system design begins with the program flow chart as shown in Fig. 5, and then makes system development combined with the division of function modules and software hierarchy.

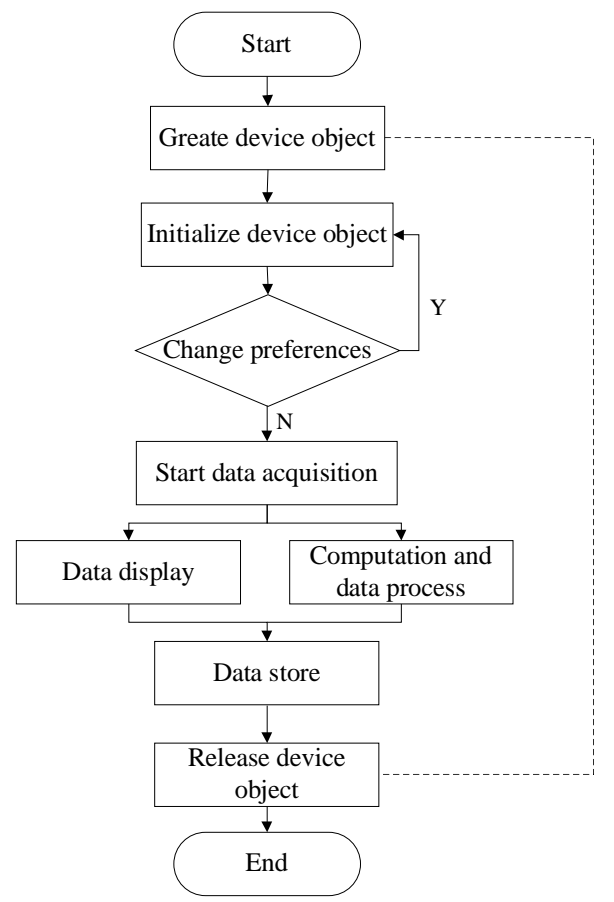

Fig. 5 the program flow chart

The LABVIEW program is shown in Fig. 6. The program mainly realizes the following functions:

1. Parameter setting: sampling device selecting, sampling voltage settings, channel settings, sample frequency settings.

2. Data collection and processing: drive sampling control, multi-channel signals extraction, decomposition of multi-channel voltage, voltage signals conversion.

3. Results analyzing: real-time display, voltage data storage, pressure values storage.

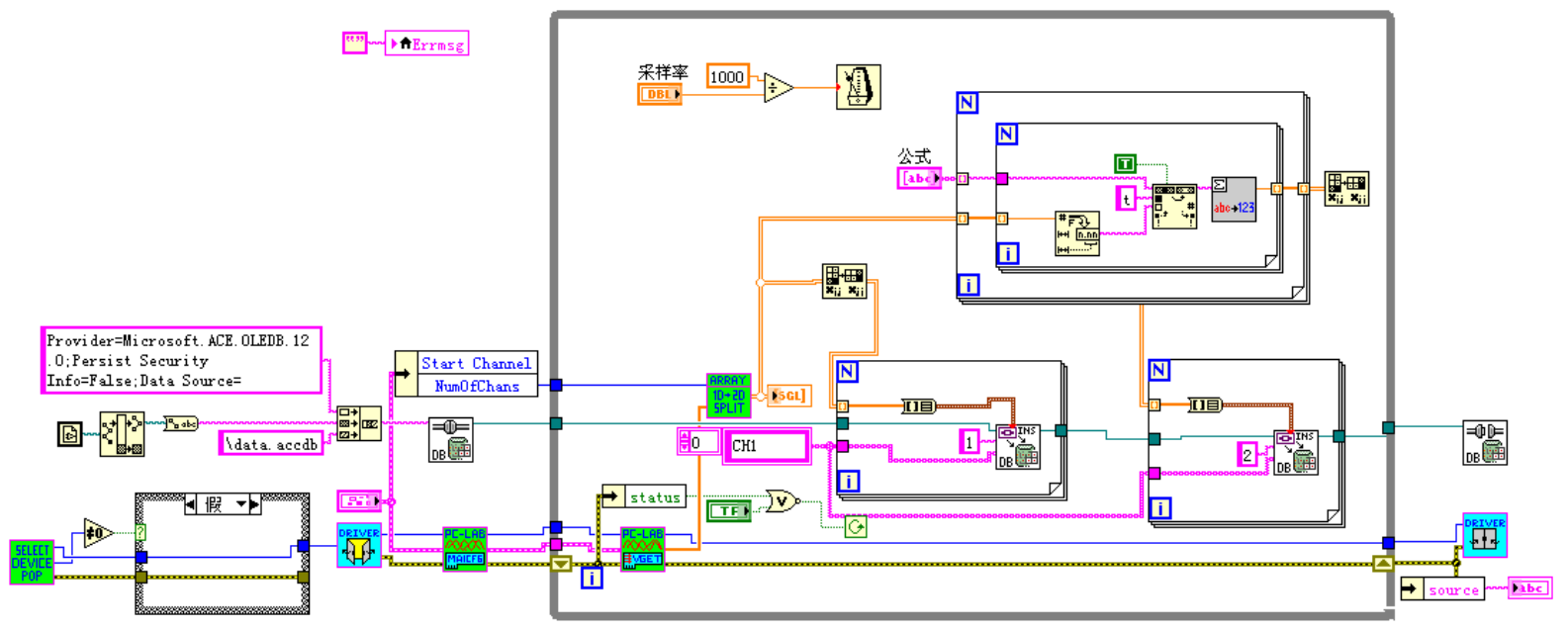

Fig. 6 The LABVIEW program 


\section{The loading experiment of the model bridge bearing}

In the Loading process, sensors are placed among rubber and steel sandwich, as shown in Fig.7. Before the sensor arrangement, drawing points in the rubber sheet, sensor number on each position is shown in Fig. 8. Sensor 1, 5, 7, 8 four peripheral points are used to measure the eccentric load of the bearing under the axial compression. Sensor 1, 2, 3, 4, 6, 7 are used to measure the stress distribution on the same straight line.

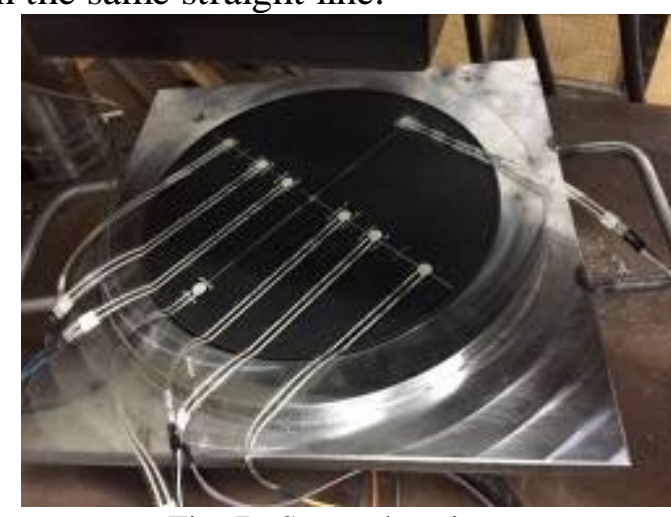

Fig. 7 Sensor locations

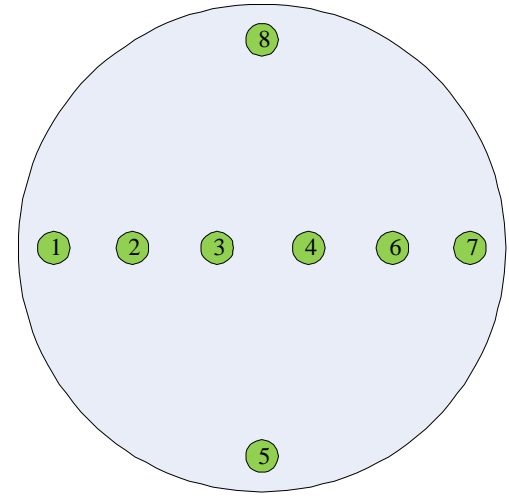

Fig. 8 Sensor number

The loading experiment of model bridge bearing simulates axial compression and eccentric compression (The loading symmetric with respect to the line sensor1 to sensor7). Two kind of operating conditions are conducted, as shown in Fig. 9 and Fig. 10.

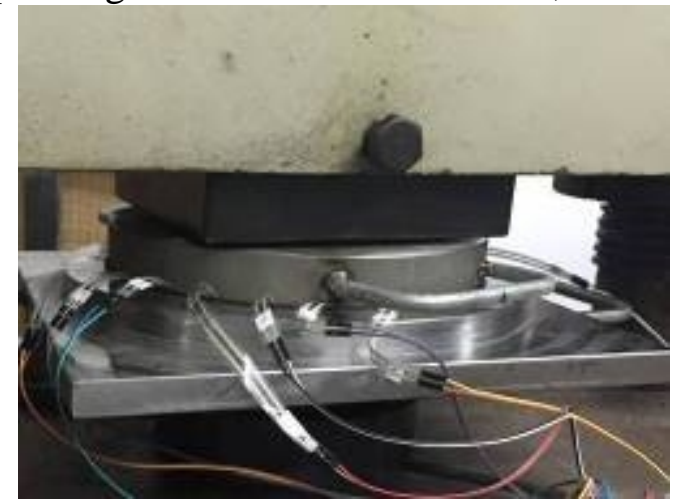

Fig. 9 Axial compression

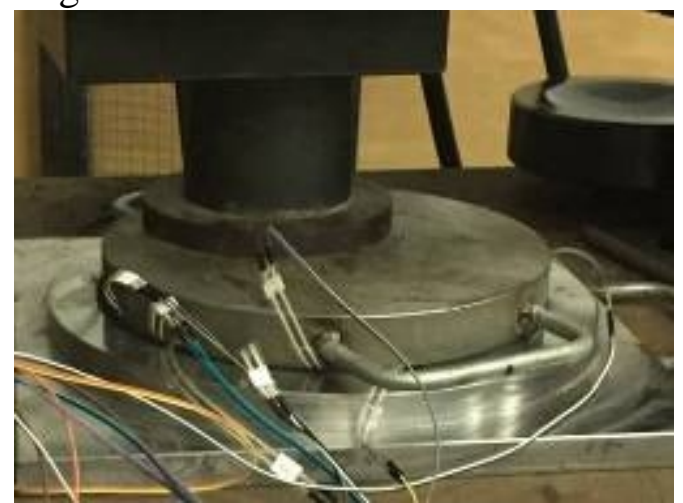

Fig. 10 Eccentric compression

In the axial compression loading test, the input load and measured load curve are shown in Fig. 11. By analyzing the data, following conclusions are drawn :

1. The input/output curve of the measuring points has good linearity, in line with the law of stress under axial compression.

2. The data about sensor 2, 3, 4, 6 show the low discrete degree, and the fitting degree of trend lines is good. The data about sensors 1, 3, 5, 7 on marginal area, with high discrete degree and the trend lines is dispersive.

3. The data have some discrepancy between measured points and the actual input load, the inner part area points show the higher measured value before $7 \mathrm{MPa}$ and lower measured value after $9 \mathrm{MPa}$. The marginal area points measured value is always lower than the input load, and with the increase of loading level the difference is more and more obvious.

The analysis of the above error: the rubber in the simple bearing model is not absolutely closed in the space. It has a deformation in the whole loading process. The deformation will cause the uneven stress distribution between the rubber and the bearing steel plates (main reflect in the marginal area of the rubber).

In the eccentric compression loading test, the input load and measured load curve are shown in Fig. 12. By analyzing the data, the following conclusions are drawn: under the condition of eccentric 
compression loading, the data about sensors $1,2,3,4,6,7$ are reduced in turn, conformed to the distribution of internal stress of bias case.

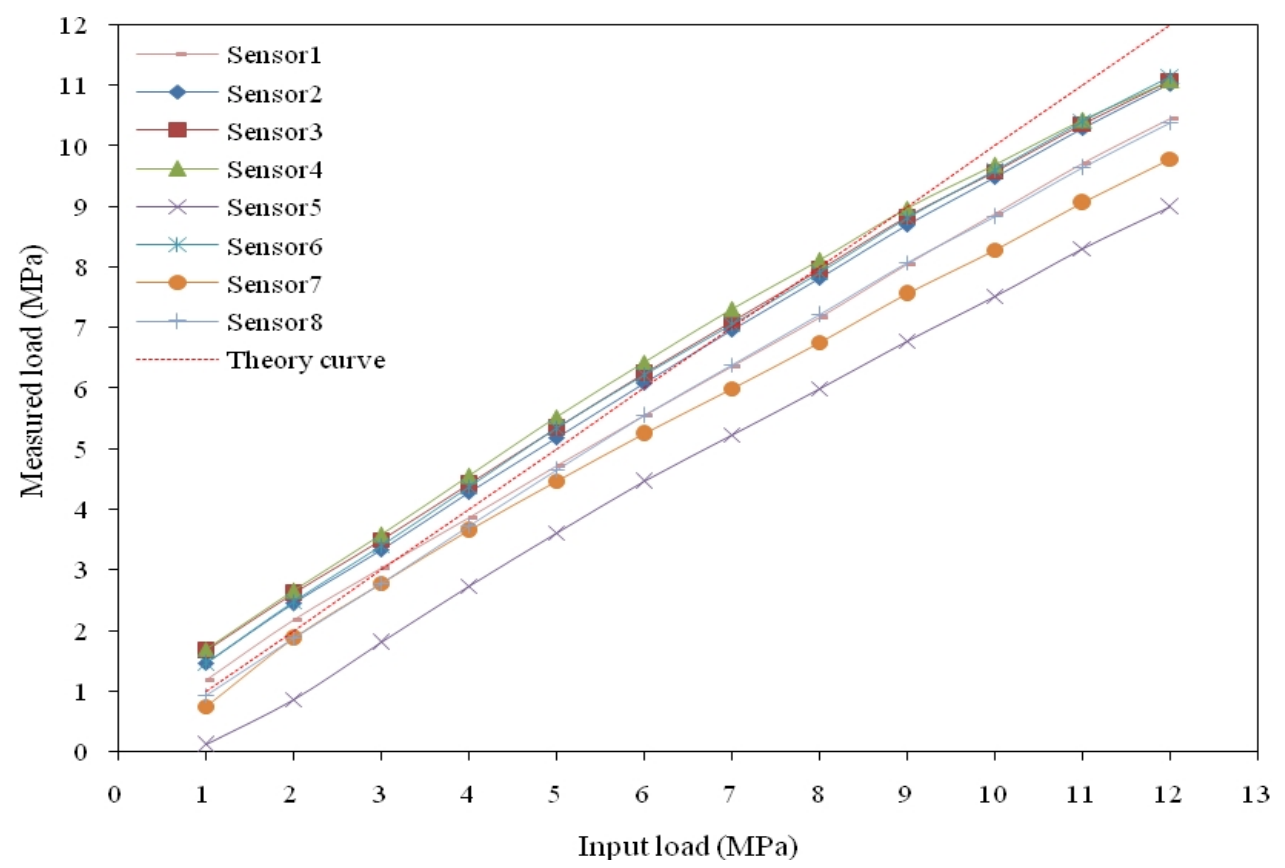

Fig. 11 The input load and measured load curve(Axial compression)

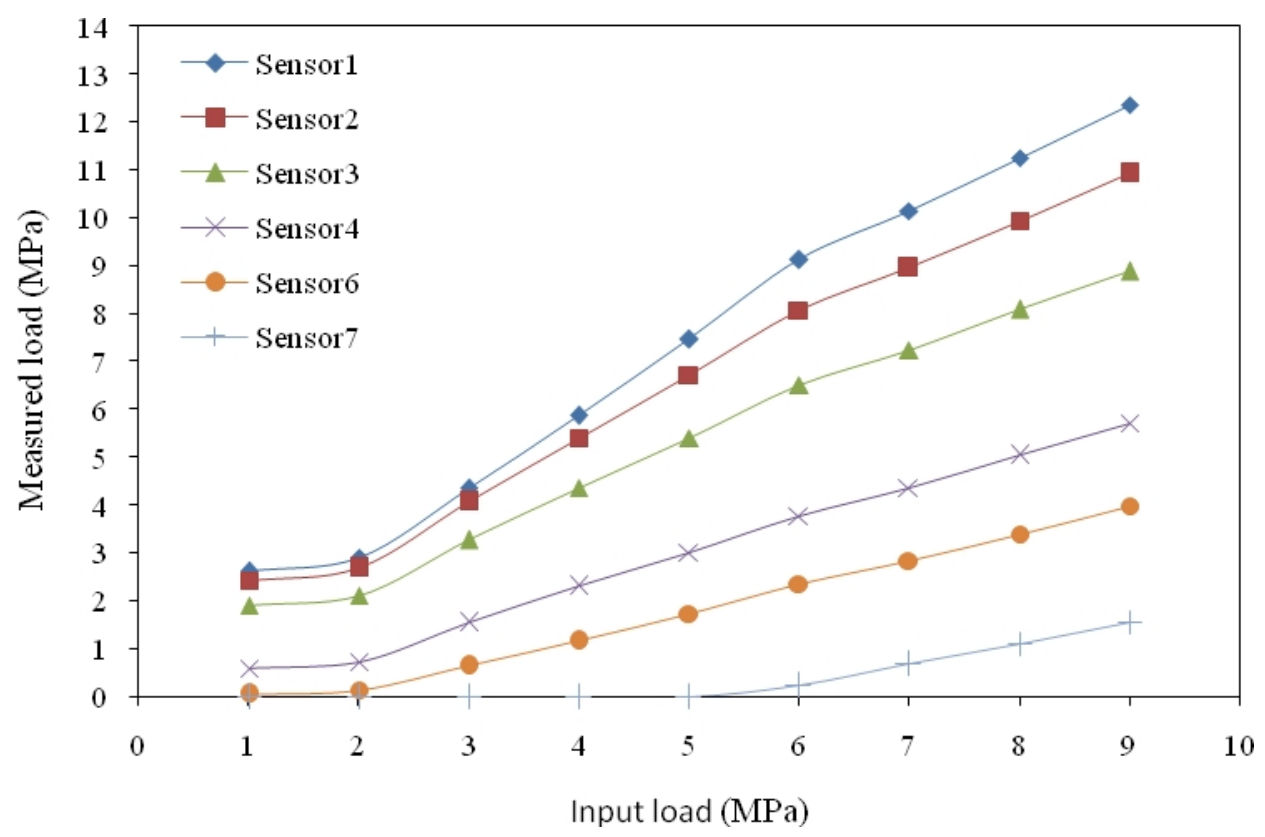

Fig. 12 The input load and measured load curve (Eccentric compression)

\section{Conclusions}

The experiments show that the proposed monitoring method for bridge bearing has promising feasibility and efficiency. This method offers an extremely high accuracy. The change trend of the monitoring data and the trend of loading under different stress state are almost the same, In a real application, the rubber is in a sealed environment, and it can reduce the error caused by the deformation of rubber. Besides, in order to achieve the higher accuracy, the secondary calibration can be applied to actual bridge bearings. 


\section{Acknowledgements}

This work was financially supported by the Science and Technology Program of Guangdong Provincial Communication Department (2014-02-014), the Key Research \& Development Plan of Shandong Province (2015GSF120003), and WEIHAI Science and Technology Development Plan.

\section{References}

[1] Ko J M, Ni Y Q. Technology developments in structural health monitoring of large-scale bridges[J]. Engineering structures, 2005, 27(12): 1715-1725.

[2] Cunha A, Caetano E, Magalhães F, et al. Recent perspectives in dynamic testing and monitoring of bridges[J]. Structural Control and Health Monitoring, 2013, 20(6): 853-877.

[3] Liu J, Miao X, Yuan Y. The rail bridge bearing monitoring system base on FBG[C].OFS2012 22nd International Conference on Optical Fiber Sensor. International Society for Optics and Photonics, 2012: 8421AQ-8421A

[4] Li S, Ning Q, Chen H. Rail Elevated Bridge Bearing Displacement Monitoring Based on FBG Sensor[J]. Applied Mechanics and Materials, 2012, 178: 2034-2037.

[5] Zang X Q, Li X B. To load three pot rubber bearing design and experimental research [J]. Railway Engineering, 2012 , (4): 1-5.

[6] Yuan P R, Li J B, Tian J D. Hydraulic call high load pot rubber bearing high performance test [J]. Road, 2009 , (10): 50-53.

[7] S. J. Chang, N. S. Kim. Development of smart seismic bridge bearing using fiber optic Bragg-grating sensors[C]. SPIE Smart Structures and Materials+ Nondestructive Evaluation and Health Monitoring.International Society for Optics and Photonics, 2013, 8692: UNSP 869228.

[8] Ha D H, Kim D, Choo J F, et al. Energy harvesting and monitoring using bridge bearing with built-in piezoelectric material[C]. IEEE The 7th International Conference on Networked Computing, 2011: 129-132.

[9] Kim J, Park Y, Choi I, et al. Development of Smart Elastomeric Bearing Equipped with PVDF Polymer Film for Monitoring Vertical Load Through the Support[J]. VDI BERICHTE, 2002, 1685: 135-140. 